\title{
EQUILIBRIUM STATES OF GRID FUNCTIONS
}

BY

\author{
NELSON G. MARKLEY AND MICHAEL E. PAUL
}

\begin{abstract}
It is well known that locally constant functions on symbolic spaces have unique equilibrium states. In this paper we investigate the nature of equilibrium states for a type of continuous function which need not have a finite range. Although most of these functions have a unique equilibrium state, phase transitions or multiple equilibrium states do occur and can be analyzed.
\end{abstract}

Introduction. The study of equilibrium states and phase transitions is a major theme of modern statistical physics. This paper examines the equilibrium states for a class of functions which have their roots in Fisher's work on condensation [1]. The nature of our paper, however, is entirely mathematical.

At each integer (site) we allow $p$ different possible behaviors, e.g. spin values or occupation numbers. The configuration space $\Omega_{p}$ consists of all bisequences on the symbols $1, \ldots, p$. Shifting the indexing one unit to the left defines a natural transformation $\sigma$ on $\Omega_{p}$. The transformation group $\left(\Omega_{p}, \sigma\right)$ is usually called the full shift on $p$-symbols. Let $X$ be a closed $\sigma$-invariant $(\sigma X=X)$ subset of $\Omega_{p}$. We will consider a class of continuous functions which are zero on $X$, have a finite range off certain open sets, and depend only on the terms with nonnegative indices in a bisequence. Because of the latter property we can delete the negatively indexed terms and work with sequences. In particular the Ruelle operator will play an important role.

The equilibrium states investigated by Hofbauer [2] fall into our general context. In his paper $X$ is the bisequence of all l's, and given a sequence the value of the function is determined by the number of consecutive l's beginning at the 0th coordinate. His work includes the existence of functions whose only equilibrium state is a point mass. Independently the authors [4] also found such examples in a preliminary investigation of the following problem: Given $X$, do there exist functions whose equilibrium states are precisely the maximal measures on $X$, i.e. the measures supported on $X$ with entropy equal to the topological entropy of $X$. This problem and the spirit of Hofbauer's results are the fountainhead of the present paper.

The initial three sections contain results for arbitrary closed subspaces. First we use Israel's Theorem to give an affirmative answer to the above problem and to show that phase transitions always occur in a space of grid functions. In particular, there are grid functions for which the equilibrium states are the closed convex hull of the

Received by the editors September 11, 1981.

1980 Mathematics Subject Classification. Primary 28D20, 54H20; Secondary 82A05. 
maximal measures and one measure supported off $X$. Because these functions vanish on $X$, the maximal measures are equilibrium states if and only if the pressure equals the topological entropy of $X$. When the pressure is greater than the topological entropy of $X$, we show that a grid function satisfies the Ruelle-Perron-Frobenius condition and hence behaves very much like a locally constant function.

The last four sections are devoted to more detailed results when $X$ is a subshift of finite type given by a $0-1$ matrix. The main idea in these sections is to exploit the connection between a grid function and an associated matrix valued analytic function of one complex variable. In $\$ 6$ we explicitly calculate the positive eigenfunction for the Ruelle operator when the pressure is greater than the topological entropy of $X$. In the last section we isolate a special class of grid functions whose pressure equals the topological entropy of $X$. When we pass through these functions in certain directions, the equilibrium states bifurcate in one of only two possible ways, which we characterize analytically. In the last three sections only the barest indication of the method of proof is given. For the reader who is interested detailed proofs can be found in our Technical Report [5].

1. Grid functions. Consider the full two-sided shift $\sigma: \Omega_{p} \rightarrow \Omega_{p^{\prime}}$ that is

$$
w=\left(w_{n}\right)_{n=-\infty}^{\infty} \in \Omega_{p}
$$

provided $w_{n} \in\{1,2, \ldots, p\}$ for each integer $n$ and $\sigma$ is defined by $(\sigma w)_{n}=w_{n+1}$. As usual $\Omega_{p}$ is given the product topology resulting from $\{1, \ldots, p\}$ being given the discrete topology. The one-sided shift $\sigma: \Omega_{p}^{+} \rightarrow \Omega_{p}^{+}$is defined similarly with typical element $w=\left(w_{n}\right)_{n=0}^{\infty}$. Given a block $B=b_{0} b_{1} \cdots b_{\text {l }}$ of symbols from $\{1, \ldots, p\}$, we shall also refer to the cylinder $B$ in $\Omega_{p}$ or $\Omega_{p}^{+}$defined by

$$
B=\left\{w: w_{0} w_{1} \cdots w_{l}=b_{0} b_{1} \cdots b_{l}\right\} \text {. }
$$

Let $\Re$ denote the set of probability measures on the Borel subsets of $\Omega_{p}$ endowed with the weak *-topology from $C\left(\Omega_{p}\right)$, the Banach space of continuous real valued functions on $\Omega_{p}$ with the supremum norm \|\|$_{\infty}$. Also let $\Re(\sigma)$ denote the $\sigma$ invariant elements of $\mathfrak{K}$, and define $\mathfrak{K}^{+}$and $\mathfrak{K}^{+}(\sigma)$ analogously for $\Omega_{p}^{+}$. By deleting the terms with negative index from points in $\Omega_{p}, C\left(\Omega_{p}^{+}\right)$may be viewed in a natural way as a closed subspace of $C\left(\Omega_{p}\right)$. It is easy to see that restricting $\mu$ in $\Re(\sigma)$ to $C\left(\Omega_{p}^{+}\right)$induces a bijection between $\Re(\sigma)$ and $\Re^{+}(\sigma)$ which preserves entropy.

If $f$ is an element of $C\left(\Omega_{p}\right)$ then the pressure of $f$, denoted by $P(f)$, is determined by

$$
P(f)=\max _{\mu \in \mathscr{R}(\sigma)}\left\{\int f d \mu+s(\mu)\right\},
$$

where $s(\mu)$ is the measure theoretic entropy of $\mu$. In other words, for our purpose it is convenient to use the variational principle to define pressure. A complete account of this principle can be found in Walters [9]. It is well known that the pressure function is convex and continuous, in fact, $|P(f)-P(g)| \leqslant\|f-g\|_{\infty}$. The set of equilibrium states for $f \in C\left(\Omega_{p}\right)$, denoted by $I(f)$, is determined by

$$
I(f)=\left\{\mu \in \mathfrak{R}(\sigma): P(f)=\int f d \mu+s(\mu)\right\} .
$$


Because $\sigma$ is expansive, $I(f) \neq \varnothing$. It is well known that if $f$ is locally constant (continuous with finite range) then $I(f)$ contains exactly one measure and this measure is positive on open sets [8].

Let $V$ be a separable Banach space and let $Q: V \rightarrow \mathbf{R}$ be a convex continuous real valued function. A linear functional $\alpha: V \rightarrow \mathbf{R}$ is called tangent to $Q$ at $x \in V$ if

$$
Q(x+y) \geqslant Q(x)+\alpha(y)
$$

for all $y \in V$. A linear functional $\alpha$ is called $Q$-bounded if there exists $c \in \mathbf{R}$ such that $\alpha-c \leqslant Q$. The following results are discussed in Ruelle [6, Appendix A.3].

I. At each $x$ in $V$ the set of tangent functionals is a nonempty subset of $V^{*}$, the dual space of $V$. In particular, each tangent functional is continuous.

II. The set of $x$ in $V$ such that there is only one tangent functional is residual (theorem of Mazur).

III. Given any $x \in V$, the set

$$
\left\{\alpha \in V^{*}: \alpha \text { is tangent to } Q \text { at } x\right\}
$$

is the closed convex hull (for the weak *-topology of the set of limits

$$
\alpha=\lim _{n \rightarrow \infty} \alpha_{n}
$$

where $\alpha_{n}$ is the only tangent functional to $Q$ at $x_{n}$ and $\lim _{n \rightarrow \infty} x_{n}=x$ (theorem of Lanford and Robinson).

IV. Given $\varepsilon>0$, if $\alpha_{0} \in V^{*}$ is $Q$-bounded and $W$ is a closed subspace of $V$, then there is $\alpha \in V^{*}$ such that $\alpha$ is tangent to $Q$ at some point $w \in W$ and such that $\left\|\left(\alpha-\alpha_{0}\right) \mid W\right\| \leqslant \varepsilon$ (theorem of Israel).

These results all apply to the pressure function $P$ and it is well known that the equilibrium states for $f$ and the tangent functionals to $P$ at $f$ coincide (see Ruelle $[6,6.14])$.

Now let $X$ be a subshift of $\Omega_{p}$, i.e. $X$ is a closed nonempty $\sigma$-invariant subset of $\Omega_{p}$. Consider a partition $\mathscr{P}=\left\{\rho(X), M_{0}, M_{1}, \ldots\right\}$ of $\Omega_{p}^{+}$satisfying the following conditions:

(a) $\rho(X)=\left\{x \in \Omega_{p}^{+}: \exists w \in X\right.$.with $\left.x=w_{0} w_{1} w_{2} \cdots\right\}$

(b) each $M_{n}$ is closed and open, $n=0,1,2, \ldots$,

(c) $\lim _{n \rightarrow \infty} M_{n}=\rho(X)$, where the limit is taken with respect to the Hausdorff metric on the closed subsets of $\Omega_{p}^{+}$,

(d) there exists $K_{0}$ such that if $B$ is any cylinder set coming from a block $B$ whose length is larger than $K_{0}$ and such that $B \cap \rho(X)=\varnothing$, then there exists $j(B)$ such that $B \subset M_{j(B)}$,

(e) for each positive integer $s$ there is a length $l_{0}(s)>K_{0}$ such that $j(B) \geqslant s$ whenever $B=B^{\prime} b_{l}=b_{0} b_{1} \cdots b_{l-1} b_{l}$ is a block with $l \geqslant l_{0}(s), B \cap \rho(X)=\varnothing$, and $B^{\prime} \cap \rho(X) \neq \varnothing$.

An example of such a partition results from setting

$$
\begin{aligned}
& M_{n}=\left\{x \in \Omega_{p}^{+}: \exists w \in X \ni w_{0} w_{1} \cdots w_{n-1}=x_{0} x_{1} \cdots x_{n-1}\right. \\
& \left.\& \nexists w \in X \ni w_{0} w_{1} \cdots w_{n}=x_{0} x_{1} \cdots x_{n}\right\} \text {. }
\end{aligned}
$$


Let $\mathcal{P}$ be any partition satisfying (a) through (e) and consider the closed subspace $\mathcal{G}(X, \mathcal{P}) \subset C\left(\Omega_{p}^{+}\right) \subset C\left(\Omega_{p}\right)$ defined by

$$
\mathcal{G}(X, \mathscr{P})=\left\{g=\sum_{n=0}^{\infty} a_{n} \chi_{M_{n}}: \lim _{n \rightarrow \infty} a_{n}=0\right\} .
$$

We will call $\mathcal{G}(X, \mathscr{P})$ the set of grid functions associated with $X$ and $\mathscr{P}$.

Throughout the rest of this paper $X$ will be a subshift of $\Omega_{p}$ and $\mathscr{P}$ will be a partition satisfying (a) through (e). Let $h(X)$ denote the topological entropy of $X$ and set $\hat{\mathscr{R}}(X)=\{\mu \in \mathfrak{N}(\sigma): \mu(X)=1 \& s(\mu)=h(X)\}$.

THEOREM 1.1. There exists $f \in \mathcal{G}(X, \mathcal{P})$ such that $P(f)=h(X)$ and $I(f)=\hat{\mathfrak{T}}(X)$.

Proof. We will apply Israel's Theorem to the pressure with $V=C\left(\Omega_{p}\right)$ and $W=\mathcal{G}(X, \mathcal{P})$. Let $\varepsilon=\frac{1}{2}$ and $\alpha_{0} \in \mathfrak{R}(\sigma)$ such that $\alpha_{0}(X)=1$. Then there exist $f \in \mathcal{G}(X, \mathcal{P})$ and $\alpha \in C\left(\Omega_{p}\right)^{*}$ such that $\alpha$ is tangent to $P$ at $f$ (in particular, $\alpha \in I(f)$ $\subset \mathfrak{R}(\sigma))$ and such that

$$
\left|\left(\alpha-\alpha_{0}\right)(g)\right| \leqslant \frac{1}{2}
$$

for all $g \in \mathcal{G}(X, \mathcal{P})$ with $\|g\|_{\infty} \leqslant 1$. First we will show that $\alpha(X)>0$. If $\alpha(X)=0$, then we can find $l$ such that $\alpha\left(\cup_{i=0}^{l} M_{i}\right)>\frac{1}{2}$. Letting $g=\sum_{i=0}^{l} \chi_{M_{i}} \in \mathcal{G}(X, \mathscr{P})$, we have the contradiction that $\|g\|_{\infty}=1$ and $\left(\alpha-\alpha_{0}\right)(g)=\alpha g>\frac{1}{2}$.

Next we show that $\hat{\mathfrak{R}}(X) \cap I(f) \neq \varnothing$. Since $I(f)$ is a Choquet simplex and $\alpha(X)>0$, the ergodic decomposition of $\alpha$ must contain an ergodic measure $\mu$ in $I(f)$ with $\mu(X)>0$. Then clearly $\mu \in \hat{\mathfrak{R}}(X)$. It follows that $P(f)=h(X)$.

Finally consider $\hat{f} \in \mathcal{G}(X, \mathcal{P})$ defined by

$$
\hat{f}=f-\sum_{i=0}^{\infty} \frac{1}{i+1} \chi_{M_{i}} .
$$

Since $\hat{f} \leqslant f, P(\hat{f}) \leqslant P(f)$. But $\hat{f}|X=0=f| X$, so the equilibrium states for $\hat{f}$ are precisely those equilibrium states for $f$ which are supported entirely on $X$. Consequently $P(\hat{f})=h(X)$ and $I(\hat{f})=\hat{\mathfrak{C}}(X)$.

THEOREM 1.2. There exists $f \in \mathcal{G}(X, \mathcal{P})$ such that $\hat{\mathfrak{R}}(X) \subset I(f)$ and $\hat{\mathfrak{R}}(X) \neq$ $I(f)$.

Proof. We again apply Israel's Theorem with $V=C\left(\Omega_{p}\right)$ and $W=\mathcal{G}(X, \mathscr{P})$. This time let $\varepsilon=\frac{1}{4}$ and let $\alpha_{0}$ be an element of $\mathscr{N}(\sigma)$ such that $\alpha_{0}(X)=\frac{1}{2}$. Then there exist $f \in \mathcal{G}(X, \mathscr{P})$ and $\alpha \in C\left(\Omega_{p}\right)^{*}$ such that $\alpha$ is tangent to $P$ at $f$ and such that

$$
\left|\left(\alpha-\alpha_{0}\right)(g)\right|<\frac{1}{4}
$$

for all $g \in \mathcal{G}(X, \mathscr{P})$ with $\|g\|_{\infty} \leqslant 1$. Choose $l$ so that $\alpha_{0}\left(\cup_{i=0}^{l}\left(M_{i}\right)\right)>\frac{1}{4}$ and set $g=\sum_{i=0}^{\prime} \chi_{M_{i}} \in \mathcal{S}(X, \mathcal{P})$. If $\alpha(X)=1$, then $\left(\alpha_{0}-\alpha\right)(g)=\alpha_{0}(g)>\frac{1}{4}$, a contradiction. If $\alpha(X)=0$, find $s$ so that $\alpha\left(\cup_{i=1}^{s} M_{i}\right)>\frac{3}{4}$ and let $h=-\sum_{i=1}^{s} \chi_{M_{i}}$. Then $\left(\alpha_{0}-\alpha\right)(h)>-\frac{1}{2}+\frac{3}{4}=\frac{1}{4}$, another contradiction. Thus $0<\alpha(X)<1$.

These theorems admit the following generalization: Let $\phi: X \rightarrow X$ be an expansive $\mathrm{Z}$-action, $X$ is a compact metric space. Let $Y \neq \varnothing$ be a closed $\phi$-invariant subset of $X$. Then there exists a continuous real valued function $f$ defined on $X$ such that: 
(i) $f \equiv 0$ on $Y$,

(ii) $P(f)=$ topological entropy of $Y$,

(iii) $I(f)=\{\mu: \mu$ is $\phi$-invariant, $\mu(Y)=1, s(\mu)=$ topological entropy of $Y\}$.

If there is a $\phi$-invariant measure not supported on $Y$, then there exists $f$ such that (i) and (ii) hold but (iii) is false.

We see from the Theorems 1.1 and 1.2 that degenerate equilibrium states and phase transition occur in $\mathcal{G}(X, \mathcal{P})$, neither of which occurs for locally constant functions. The goal of this paper is to understand the occurrence of these phenomena in $\mathcal{G}(X, \mathcal{P})$.

2. Eigenmeasures. Given $g \in C\left(\Omega_{p}^{+}\right)$, define the Ruelle operator $\mathcal{L}=\mathcal{L}_{g}: C\left(\Omega_{p}^{+}\right)$ $\rightarrow C\left(\Omega_{p}^{+}\right)$by

$$
(\varrho f)(x)=\sum_{y \in \sigma^{-1} x} e^{g(y)} f(y)
$$

where $\sigma$ denotes the one-sided shift $\sigma: \Omega_{p}^{+} \rightarrow \Omega_{p}^{+},(\sigma x)_{n}=x_{n+1}, n \geqslant 0$. Applying the Schauder-Tychonoff fixed point theorem to the continuous map $\mu \rightarrow \complement^{*} \mu /\left(\varrho^{*} \mu\right)(1)$ of $\Re^{+}$into $\Re^{+}$yields $\nu \in \Re^{+}$and $\lambda>0$ such that $\mathcal{L}^{*} \nu=\lambda \nu$ and we will call such a measure $\nu$ an eigenmeasure.

Proposition 2.1. Suppose $\nu$ is an eigenmeasure.

(i) If $A$ is open or closed in $\Omega_{p}^{+}$and satisfies $\sigma^{-1}(\sigma(A))=A$, then $\nu(A)=0$ if and only if $\nu(\sigma(A))=0$.

(ii) If $A$ is a nonempty open set, then $\nu(A)>0$.

(iii) If $F$ is a closed subset of $\Omega_{p}^{+}$and $\nu(F)=0$, then $\nu\left(\sigma^{-k}(F)\right)=0$ for all $k \geqslant 0$.

(iv) If $F$ is a closed subset of $\Omega_{p}^{+}$such that $\sigma(F) \subset F$ and $\nu(F)=0$, then $\nu\left(\sigma^{\prime}(F)\right)=0$ for all $l$.

Proof. (i) Let $E_{j}=\left\{x: x_{0}=j\right\}$ for $1 \leqslant j \leqslant p$. Then $\chi_{A \cap E_{j}}$ is the pointwise limit of continuous functions and thus

$$
\lambda \nu\left(A \cap E_{j}\right)=\lambda \int \chi_{A \cap E_{j}} d \nu=\int e \chi_{A \cap E_{j}} d \nu=\int e^{g(j x)} \chi_{\sigma A}(x) d \nu
$$

because $j x \in A$ if and only if $x \in \sigma A$. Therefore $\nu\left(A \cap E_{j}\right)=0$ if and only if $\nu(\sigma A)=0$.

(ii) Without loss of generality we assume that $A$ is a cylinder. Let $B$ be any cylinder such that $\nu(B)>0$. Define $A B=\left\{x: x \in A\right.$ and $\left.\sigma^{s} x \in B\right\}$, where $s$ is the length of $A$. Then

$$
\lambda^{s} \nu(A B)=\lambda^{s} \int \chi_{A B} d \nu=\int \mathcal{L}^{s} \chi_{A B}(x) d \nu,
$$

where the integrand is positive on the set $B$. Thus $\nu(A) \geqslant \nu(A B)>0$.

(iii) Let $A=\sigma^{-1}(F)$ and use (i).

(iv) This follows immediately from (iii) and the proof is finished.

Proposition 2.2. Let $f \in C\left(\Omega_{p}^{+}\right)$. Then there is an eigenmeasure $\nu \in \mathfrak{R}^{+}$such that $\mathcal{L}_{f}^{*} \nu=e^{P(f)} \nu$.

Proof. If $f$ were locally constant, then this proposition would follow from the Perron-Frobenius Theorem. A proof which covers the case that $f$ is locally constant 
can be found in Walters [9]. Given an arbitrary $f \in C\left(\Omega_{p}^{+}\right)$, let $f_{n}$ be a sequence of locally constant functions converging in the supremum norm to $f$, and let $\lambda_{n}=e^{P\left(f_{n}\right)}$. Since pressure is a continuous function on $C\left(\Omega_{p}^{+}\right)$, it follows that $\lim \lambda_{n}=\lambda=e^{P(f)}$. For each $n$, let $\nu_{n} \in \mathfrak{R}^{+}$satisfy $\mathcal{L}_{f_{n}}^{*} \nu_{n}=\lambda_{n} \nu_{n}$, and without losss of generality suppose that the $\nu_{n}$ converge in $\Re^{+}$to $\nu$. To see that $\mathcal{L}_{f}^{*} \nu=\lambda \nu$, let $h \in C\left(\Omega_{p}^{+}\right)$. Then

$$
\begin{aligned}
\left|\int \varrho_{f} h d \nu-\lambda \int h d \nu\right| & =\lim _{n \rightarrow \infty}\left|\int \varrho_{f} h d \nu_{n}-\lambda \int h d \nu_{n}\right| \\
& \leqslant \lim _{n \rightarrow \infty}\left|\int \varrho_{f} h d \nu_{n}-\int \varrho_{f_{n}} h d \nu_{n}\right|+\lim _{n \rightarrow \infty}\left|\int \varrho_{f_{n}} h d \nu_{n}-\lambda \int h d \nu_{n}\right| \\
& =\lim _{n \rightarrow \infty}\left|\int\left(\varrho_{f} h-\varrho_{f_{n}} h\right) d \nu_{n}\right|+\lim _{n \rightarrow \infty}\left|\lambda_{n}-\lambda\right|\left|\int h d \nu_{n}\right| \\
& \leqslant \lim _{n \rightarrow \infty}\left\|\varrho_{f} h-\varrho_{f_{n}} h\right\|_{\infty}+\left|\int h d \nu\right| \lim _{n \rightarrow \infty}\left|\lambda_{n}-\lambda\right| \\
& =0+0 .
\end{aligned}
$$

The next lemma will be used in Theorem 2.4 and also in $\$ 3$.

LEMMA 2.3. Let $g \in \mathcal{G}(X, \mathcal{P})$ and let $E$ be any cylinder of length $N>K_{0}$ (where $K_{0}$ comes from condition (d) on the partition $\mathcal{P}$ ) such that $E \cap \rho(X)=\varnothing$. Now let $B$ be any cylinder with length $l$. If $x$ and $y$ in $\Omega_{p}^{+}$agree in coordinates 0 through $N-1$, then $\mathfrak{L}_{g}^{l} \chi_{B E}(x)=\mathfrak{L}_{g}^{l} \chi_{B E}(y)$. Thus we may define a constant $K(B, E)$ by choosing any point $z \in E$ and setting $K(B, E)=\mathcal{L}_{g} \chi_{B E}(z)$. Furthermore, if $\mathcal{L}_{g}^{*} \nu=\lambda \nu$, then $\nu(B E)=$ $\lambda^{-l} K(B, E) \nu(E)$.

Proof. That $\mathcal{L}^{l} \chi_{B E}(x)=\mathscr{L}^{l} \chi_{B E}(y)$ follows from $E$ having length larger that $K_{0}$. Also,

$$
\nu(B E)=\int \chi_{B E} d \nu=\frac{1}{\lambda^{\prime}} \int \mathcal{L}^{\prime} \chi_{B E} d \nu=\frac{1}{\lambda^{\prime}} K(B, E) \nu(E) .
$$

The main result of this section is the following:

THEOREM 2.4. Let $g \in \mathcal{G}(X, \mathcal{P})$. If $P(g)>h(X)$, then there is exactly one $\nu \in \mathfrak{R}^{+}$ such that $\mathcal{L}^{*} \nu=e^{P(g)} \nu$.

Proof. Proposition 2.2 asserts the existence of at least one such $\nu \in \mathfrak{R}^{+}$. We need to show that $\nu$ is unique. Let $\lambda=e^{P(g)}$ and choose $\gamma$ such that $e^{h(X)}=\alpha<\gamma<$ $\lambda$. The definition of topological entropy then insures the existence of a positive constant $K$ such that for all $n$, the number of $n$-blocks appearing in $X$ is less than $K \gamma^{n}$. Therefore

$$
\begin{aligned}
\nu(\rho(X)) & =\int \chi_{\rho(X)}(z) d \nu=\frac{1}{\lambda^{n}} \int \varrho^{n} \chi_{\rho(X)}(z) d \nu \\
& \leqslant K\left(\frac{\gamma}{\lambda}\right)^{n} \nu(\rho(X)) .
\end{aligned}
$$

Since $\gamma / \lambda<1$ and $n$ is arbitrary, it follows that $\nu(\rho(X))=0$, and then by Proposition 2.1 that $\nu\left(\sigma^{\prime} \rho(X)\right)=0$ for all integers $l$. Now choose $0<\varepsilon<1$ such that 
$\varepsilon \lambda / \gamma>1$ and then choose $N_{0}>K_{0}$ (where $K_{0}$ comes from condition (d) on the partition $\mathcal{P}$ ) so that:

(i) The subshift of finite type $\bar{X}$ determined by the $N_{0}$-blocks appearing in $X$ has topological entropy less than $\log \gamma$.

(ii) If $B=b_{0} b_{1} \cdots b_{l}$ is any cylinder set whose length is larger than or equal to $N_{0}$ with $B \cap \rho(X)=\varnothing$ and $b_{0} b_{1} \cdots b_{l-1} \cap \rho(X) \neq \varnothing$ then $\exp \left(a_{j(B)}\right)<\varepsilon \lambda / \gamma$.

Let $K$ now be any constant such that the number of $n$-blocks in $X$ is less than $K \gamma^{n}$ for all $n$. Conditions (i) and (ii) imply that for $y \in \bar{X}, e^{g(y)}<\varepsilon \lambda / \gamma$ and thus

$$
\begin{aligned}
\nu(\bar{X}) & =\int \chi_{\bar{X}}(z) d \nu=\frac{1}{\lambda^{n}} \int \mathcal{L}^{n} \chi_{\bar{X}}(z) d \nu \\
& \leqslant \frac{K \gamma^{n}}{\lambda^{n}}\left(\frac{\varepsilon \lambda}{\gamma}\right)^{n} \nu(\bar{X})=K \varepsilon^{n} \nu(\bar{X}) .
\end{aligned}
$$

Therefore $\nu(\bar{X})=0$. Now let

$$
C=\left\{w \in \Omega_{p}^{+}: w_{0} \cdots w_{N_{0}-1} \text { does not appear in } \bar{X}\right\} .
$$

Then $\nu\left(\cup_{l=0}^{\infty} \sigma^{-l} \bar{X}\right)=0$ implies that the set $S=\left\{w \in \Omega_{p}^{+}: \sigma^{n_{i}} w \cap C \neq \varnothing\right.$ for infinitely many positive integers $\left.n_{i}\right\}$ must satisfy $\nu(S)=1$. Suppose now that $B=b_{0} b_{1} \cdots b_{l-1}$ is any cylinder set. Let $C=\cup_{i=1}^{L} C_{i}$ where each $C_{i}$ is an $N_{0}$-block. Applying Lemma 2.3 to each cylinder $B C_{i}$ shows that $\nu\left(B C_{i}\right)$ is determined by $\mathscr{P}, g$, and $\nu\left(C_{i}\right)$. But $\nu(S)=1$ implies that every cylinder set $B$ can be written as the countable disjoint union of sets of the form $B D C_{i}$, along with a set of $\nu$-measure zero. Thus $\nu$ would be uniquely determined if the numbers $\left\{\nu\left(C_{i}\right)\right\}_{i=1}^{L}$ were known. Proposition 2.1 implies that $\nu$ assigns positive measure to each cylinder $C_{i}$. But

$$
C_{1}=C_{1} C_{1} \cup C_{1} C_{2} \cup \cdots \cup C_{1} C_{L} \cup \cdots \cup C_{1} D C_{j} \cup \cdots,
$$

where each $D$ is a block containing no $C_{j}$ as a sub-block, and the equality is true up to a set of $\nu$-measure zero. We then have the following set of equations:

$$
\begin{gathered}
\nu\left(C_{1}\right)=K_{11} \nu\left(C_{1}\right)+K_{12} \nu\left(C_{2}\right)+\cdots+K_{1 L} \nu\left(C_{L}\right) \\
\vdots \\
\nu\left(C_{L}\right)=K_{L 1} \nu\left(C_{1}\right)+K_{L 2} \nu\left(C_{2}\right)+\cdots+K_{L L} \nu\left(C_{L}\right),
\end{gathered}
$$

where each $K_{i j}$ is a positive constant determined by $\mathcal{P}$ and $g$. Thus the vector $V=\left(\nu\left(C_{1}\right), \nu\left(C_{2}\right), \ldots, \nu\left(C_{L}\right)\right)$ is a strictly positive eigenvector of the irreducible matrix $\left(K_{i j}\right)$. Therefore $V$ is determined up to a scalar. But we may decompose $\Omega_{p}^{+}$ into sets of the form $A C_{i}$, and therefore $\nu\left(\Omega_{p}^{+}\right)=1=\sum_{i=1}^{L} e_{i} \nu\left(C_{i}\right)$, where the $e_{i}$ are constants determined by $\mathcal{P}$ and $g$. Therefore $V$ is determined solely by $\mathcal{P}$ and $g$, and so $\nu$ is unique.

3. Characterization of RPF grid functions. Given $g \in C\left(\Omega_{p}^{+}\right)$, one says that $g$ satisfies the Ruelle-Perron-Frobenius (RPF) condition provided that there exists $\lambda>0, H \in C\left(\Omega_{p}^{+}\right)$with $H>0$ and $\nu \in \mathfrak{N}^{+}$such that $\mathcal{L}_{g} H=\lambda H, \mathcal{L}_{g}^{*} \nu=\lambda \nu$, $\int H d \nu=1$, and

$$
\lim _{n \rightarrow \infty}\left\|\lambda^{-n} \stackrel{L}{g}_{g}^{n}(f)-\left(\int f d \nu\right) H\right\|_{\infty}=0
$$

for all $f$ in $C\left(\Omega_{p}^{+}\right)$. The following theorem appears in Hofbauer [2]. 
THEOREM. Let $g \in C\left(\Omega_{p}^{+}\right)$satisfy the RPF condition. Then $\mu=H \nu$ is the only o-invariant measure on $\Omega_{p}^{+}$such that $P(g)=\int g d \mu+s(\mu)$. Thus there is only one measure $\tilde{\mu}$ in $I(g)$ and $\tilde{\mu}$ restricted to $C\left(\Omega_{p}^{+}\right)$equals $\mu$.

The principal goal of this section is to establish that for $g \in \mathcal{S}(X, \mathcal{P}), g$ satisfies RPF if and only if $P(g)>h(X)$. We shall also describe the structure of $I(g)$ when the grid function $g$ satisfies $P(g)=h(X)$.

Proposition 3.1. Suppose that $g \in \mathcal{G}(X, \mathcal{P})$ and $P(g)>h(X)$. Then $\left\{\lambda^{-n} \mathfrak{L}^{n} 1\right.$ : $n \geqslant 1\}$ is a uniformly bounded and equicontinuous family of functions.

Proof. We shall use the same $N_{0}$ and $C$ as in the proof of Theorem 2.4. Given $x \in \Omega_{p}^{+}$and $n \geqslant 0$, partition $\sigma^{-n} x$ as follows:

$$
\begin{gathered}
D_{0}=\left\{z \in \sigma^{-n} x: \sigma^{n} z \in C\right\}, \\
D_{1}=\left\{z \in \sigma^{-n} x: \sigma^{n-1} z \in C\right\}-D_{0}, \\
\vdots \\
D_{j}=\left\{z \in \sigma^{-n} x: \sigma^{n-j} z \in C\right\}-\bigcup_{i=0}^{j-1} D_{i}, \\
\vdots \\
D_{n}=\left\{z \in \sigma^{-n} x: z \in C\right\}-\bigcup_{j=0}^{n-1} D_{j}, \\
D_{n+1}=\left\{z \in \sigma^{-n} x: z \notin \bigcup_{j=0}^{n} D_{j}\right\} .
\end{gathered}
$$

Let $M$ be the maximum of $\exp \left(\|g\|_{\infty}\right)$ and the numbers $\left\|\mathcal{L}^{i} 1 / \lambda^{i}\right\|_{\infty}, 1 \leqslant i \leqslant N_{0}$, and let $n$ be any positive integer larger than $N_{0}$. Then for all $N_{0}<i<n+1$ the following estimate holds:

$$
\frac{1}{\lambda^{n}} \sum_{z \in D_{i}} e^{g(z)+g(\sigma z)+\cdots+g\left(\sigma^{n-1} z\right)} \leqslant K L M \frac{\nu\left(\sigma^{-(n-i)} \bar{C}\right)}{\nu(\bar{C})} \frac{\gamma^{i-N_{0}}}{\lambda^{i}}\left(\frac{\varepsilon \lambda}{\gamma}\right)^{i-1}
$$

where $\bar{C}$ is chosen from $\left\{C_{j}\right\}_{j=1}^{L}$ so as to maximize $\nu\left(\sigma^{-(n-i)} C_{j}\right) / \nu\left(C_{j}\right)$. (For $i=n+1$, the right-hand side of (*) can be replaced by $K \varepsilon^{n}$.) To see that (*) is valid, let $z$ be a typical point in $D_{i}$. Then $z$ has the form $z=A C_{j} B x$, where $A$ has length $n-i, C_{j}$ comes from the set $\left\{C_{t}\right\}_{t=1}^{L}$, and $B$ has length $i-N_{0}$. In addition, $B$ has the property that the only sub-block of $C_{j} B x_{0} x_{1} \cdots x_{N_{0}-1}$ which comes from $\left\{C_{t}\right\}_{t=1}^{L}$ is the initial block $C_{j}$. Then by Lemma 2.3

$$
e^{g\left(A C_{j} B x\right)+g\left(\sigma A C_{j} B x\right)+\cdots+g\left(\sigma^{n-i-1} A C_{j} B x\right)}=\lambda^{n-i} \frac{\nu\left(A C_{j}\right)}{\nu\left(C_{j}\right)}
$$

and also

$$
e^{g\left(\sigma^{n-i} A C_{j} B x\right)+\cdots+g\left(\sigma^{n-1} A C_{j} B x\right)} \leqslant e^{\|g\|_{\infty}}\left(\frac{\varepsilon \lambda}{\gamma}\right)^{i-1} \leqslant M\left(\frac{\varepsilon \lambda}{\gamma}\right)^{i-1} .
$$


Thus

$$
\frac{1}{\lambda^{n}} e^{g(z)+g(\sigma z)+\cdots+g\left(\sigma^{n-1} z\right)} \leqslant \frac{1}{\lambda^{i}} \frac{\nu\left(A C_{j}\right)}{\nu\left(C_{j}\right)} M\left(\frac{\varepsilon \lambda}{\gamma}\right)^{i-1} .
$$

Now fix $C_{j}$ and $B$ but let $A$ vary over all $(n-i)$ blocks:

$$
\frac{1}{\lambda^{n}} \sum_{A} e^{g\left(A C_{j} B x\right)+\cdots+g\left(\sigma^{n-1} A C_{j} B x\right)} \leqslant \frac{1}{\lambda^{i}} \frac{\nu\left(\sigma^{-(n-i)} C_{j}\right)}{\nu\left(C_{j}\right)} M\left(\frac{\varepsilon \lambda}{\gamma}\right)^{i-1} .
$$

The condition satisfied by the block $C_{j} B x_{0} x_{1} \cdots x_{N_{0}-1}$ implies in particular that $B$ must appear in the subshift of finite type $\bar{X}$, hence the number of possible choices for $B$ is less than $K \gamma^{i-N_{0}}$. Therefore,

$$
\frac{1}{\lambda^{n}} \sum_{A} \sum_{B} e^{g\left(A C_{j} B x\right)+\cdots+g\left(\sigma^{n-1} A C_{j} B x\right)} \leqslant \frac{K \gamma^{i-N_{0}}}{\lambda^{i}} \frac{\nu\left(\sigma^{-(n-i)} C_{j}\right)}{\nu\left(C_{j}\right)} M\left(\frac{\varepsilon \lambda}{\gamma}\right)^{i-1}
$$

and since $C_{j}$ comes from the set $\left\{C_{t}\right\}_{t=1}^{L}$ we have shown that the estimate (*) holds.

Now let $C^{\prime}$ denote the maximum value of $\left\{\nu\left(C_{j}\right)^{-1}\right\}_{j=1}^{L}$. Then for $i>N_{0}$ :

$$
\frac{1}{\lambda^{n}} \sum_{z \in D_{i}} e^{g(z)+\cdots+g\left(o^{n-1} z\right)} \leqslant\left(K L M C^{\prime} \gamma^{-\left(N_{0}-1\right)} \lambda\right) \varepsilon^{i-1} \text {. }
$$

For $i=0$ :

$$
\frac{1}{\lambda^{n}} \sum_{z \in D_{0}} e^{g(z)+\cdots+g\left(\sigma^{n-1} z\right)}=\sum_{A} \frac{\nu\left(A C_{j}\right)}{\nu\left(C_{j}\right)} \leqslant C^{\prime}
$$

where $A$ ranges over all possible $n$-blocks and $C_{j}$ is the initial $N_{0}$-block of $x$.

For $1 \leqslant i \leqslant N_{0}$ : Suppose $z \in D_{i}$. Then $z$ has the form $z=A \bar{C}_{j} x$, where $\bar{C}_{j}$ denotes an initial segment of $C_{j}$. As before, let $A$ range over all possible blocks and then let $\bar{C}_{j}$ range over its at most $L$ possibilities to conclude that

$$
\frac{1}{\lambda^{n}} \sum_{z \in D_{i}} e^{g(z)+\cdots+g\left(\sigma^{n-1} z\right)} \leqslant C^{\prime} L M .
$$

Therefore,

$$
\begin{aligned}
\frac{\varrho^{n} 1(x)}{\lambda^{n}} & =\sum_{i=0}^{n+1}\left(\frac{1}{\lambda^{n}} \sum_{z \in D_{i}} e^{g(z)+\cdots+g\left(\sigma^{n-1} z\right)}\right) \\
& \leqslant C^{\prime}+N_{0} C^{\prime} L M+\left(K L M C^{\prime} \gamma^{-\left(N_{0}-1\right)} \lambda\right) \sum_{i=N_{0}+1}^{\infty} \varepsilon^{i-1}
\end{aligned}
$$

Since $0<\varepsilon<1,\left\{\lambda^{-n} \complement^{n} 1: n \geqslant 1\right\}$ is uniformly bounded.

To establish equicontinuity, take any two points $x$ and $y$ in $\Omega_{p}^{+}$and suppose that they share their initial $N_{0}$-blocks. Given any $n$, form the partitions of $\sigma^{-n} x$ and $\sigma^{-n} y$ and note that

$$
\text { card } D_{i}(x)=\text { card } D_{i}(y), \quad 0 \leqslant i \leqslant n+1
$$


For $i>N_{0}$ :

$$
\begin{aligned}
& \left|\frac{1}{\lambda^{n}} \sum_{z \in D_{i}(x)} e^{g(z)+\cdots+g\left(\sigma^{n-1} z\right)}-\frac{1}{\lambda^{n}} \sum_{\bar{z} \in D_{i}(y)} e^{g(\bar{z})+\cdots+g\left(\sigma^{n-1} \bar{z}\right)}\right| \\
& \quad \leqslant 2 \max \left\{\frac{1}{\lambda^{n}} \sum_{z \in D_{i}(x)} e^{g(z)+\cdots+g\left(\sigma^{n-1} z\right)}, \frac{1}{\lambda^{n}} \sum_{\bar{z} \in D_{i}(y)} e^{g(\bar{z})+\cdots+g\left(\sigma^{n-1} \bar{z}\right)}\right\} \\
& \leqslant 2\left(K L M C^{\prime} \gamma^{-\left(N_{0}-1\right)} \lambda\right) \varepsilon^{i-1} .
\end{aligned}
$$

Therefore, given $\delta>0$, choose $N(\delta)$ so that

$$
\left(2 K L M C^{\prime} \gamma^{-\left(N_{0}-1\right)} \lambda\right) \sum_{i=N(\delta)}^{\infty} \varepsilon^{i-1}<\frac{\delta}{2} .
$$

Now use uniform continuity to find $N_{1}(\delta) \geqslant N_{0}$ such that for $x$ and $y$ with the same initial $N_{1}(\delta)$-block, the finite set of differences of the form

$$
\frac{1}{\lambda^{s}}\left|e^{g(B x)+g(\sigma B x)+\cdots+g\left(\sigma^{s-1} B x\right)}-e^{g(B y)+g(\sigma B y)+\cdots+g\left(\sigma^{s-1} B y\right)}\right|
$$

are all less than $\delta / 2 C^{\prime} N(\delta) p^{N(\delta)}$, where $B$ is any block of length $s$, with $s \leqslant N(\delta)$. This establishes the equicontinuity of the family $\left\{\lambda^{-n} \mathcal{L}^{n} 1: n \geqslant 1\right\}$.

COROLlary 3.2. Let $f \in C\left(\Omega_{p}^{+}\right)$. Then the family $\left\{\lambda^{-n} \mathcal{L}^{n} f: n \geqslant 1\right\}$ is a uniformly bounded and equicontinuous family of functions.

Proof. We assume first that $f$ is the characteristic function of some cylinder $B$, say $B=b_{0} b_{1} \cdots b_{t}$. Now redefine the subsets $\left\{D_{i}\right\}_{i=0}^{n+1}$ of $\sigma^{-n} x$ by further demanding that for all $i, z \in D_{i}$ implies $z_{0} z_{1} \cdots z_{t}=B$. Call this new collection $\left\{\bar{D}_{i}\right\}$ and then carry through the same proof as in Proposition 3.1 to show that $\left\{\lambda^{-n} \mathcal{L}^{n} f\right\}$ is bounded and equicontinuous. The truth of Corollary 3.2 for characteristic functions of cylinders implies the truth for locally constant functions. Now let $f$ be any continuous function and let $\delta>0$ be given. Choose a locally constant function $\bar{f}$ such that $\|\bar{f}-f\|_{\infty}<\delta$. Then

$$
\left|\frac{\mathfrak{e}^{n} f(x)}{\lambda^{n}}-\frac{\mathfrak{e}^{n} \bar{f}(x)}{\lambda^{n}}\right| \leqslant\|f-\bar{f}\|_{\infty} \frac{\mathfrak{e}^{n} 1(x)}{\lambda^{n}} \leqslant \delta \frac{\mathfrak{E}^{n} 1(x)}{\lambda^{n}} .
$$

Since $\lambda^{-n} \complement^{n} 1$ is bounded and the corollary is known to be true for $\left\{\lambda^{-n} \varrho^{n} \bar{f}\right\}$, it follows from the preceding inequalities that it must also hold for $\left\{\lambda^{-n} \mathcal{L}^{n} f\right\}$.

Proposition 3.3. Suppose $g \in \mathcal{G}(X, \mathcal{P})$ and $P(g)>h(X)$. Then there exists $H \in$ $C\left(\Omega_{p}^{+}\right)$such that $H>0$ and $\mathcal{L} H=\lambda H$. Furthermore, $H$ is constant on any cylinder $B$ whose length is larger than $K_{0}$ and such that $B \cap \rho(X)=\varnothing$.

REMARK 3.4. The existence of such a function $H>0$ can be shown to imply its uniqueness up to scalar multiples by using a device of Hofbauer [2]: namely, define $\hat{g}=g+\log H-\log H \circ \sigma-\log \lambda$. Then $\hat{g}$ is in a class of functions discussed by Walters [8], and his results guarantee the uniqueness of $H$. 
Proof of Proposition 3.3. Since $\left\{\lambda^{-n} \complement^{n} 1\right\}$ is bounded and equicontinuous, it follows that the family

$$
\left\{1, \frac{1+\lambda^{-1} \varrho 1}{2}, \frac{1+\lambda^{-1} \varrho 1+\lambda^{-2} \varrho^{2} 1}{3}, \ldots\right\}
$$

is also bounded and equicontinuous. Thus there exist $n_{i}$ and $H$ in $C\left(\Omega_{p}^{+}\right)$such that

$$
\left\|\frac{1+\lambda^{-1} \varrho 1+\cdots+\lambda^{-n_{1} \varrho 1}}{1+n_{i}}-H\right\|_{\infty} \rightarrow 0
$$

as $i \rightarrow \infty$, and a standard argument then shows that

$$
\varrho H(x)=\lambda H(x) \text {. }
$$

Since $\mathcal{L}^{*} \nu=\lambda \nu$ and $\nu(1)=1$, it follows that $\nu(H)=1$, and therefore that there is at least one $x$ such that $H(x)>0$. But by uniform continuity there must be some block $B$ such that $H>0$ on all points beginning with $B$, and therefore for any point $y$ we could find $N$ big enough so that $H(y)=\lambda^{-N}{ }^{N} H(y)>0$. Thus $H>0$. For every positive integer $n, \complement^{n} 1$ is constant on any cylinder $B$ such that the length of $B$ is larger than $K_{0}$ and such that $B \cap \rho(X)=\varnothing$. Thus $H$ also has this property.

Proposition 3.5. Suppose $g \in \mathcal{G}(X, \mathcal{P})$ and $P(g)>h(X)$. Let $f \in C\left(\Omega_{p}^{+}\right)$. Then $\lambda^{-n} \mathcal{L}^{n} f$ converges uniformly to $\nu(f) H$.

Proof. Let $\hat{g}=g+\log H-\log H \circ \sigma-\log \lambda$. Then $\mathcal{L}_{\hat{g}}^{n} f=\left(1 / \lambda^{n} H\right) \varrho^{n} H f$, and so by Corollary 3.2 one sees that $\left\{\mathcal{L}_{\hat{g}}^{n} f\right\}_{n=1}^{\infty}$ is bounded and equicontinuous. Walters [8], using an argument of Keane, shows that $\left\{\mathcal{L}_{\hat{g}}^{n} f\right\}$ equicontinuous implies that $\varrho_{\hat{g}}^{n} f$ converges to $\nu(H \dot{f})$ in $C\left(\Omega_{p}^{+}\right)$. Thus $\lambda^{-n} \mathscr{L}^{n} f$ converges to $\nu(f) H$ and the proof is finished.

THEOREM 3.6. Suppose $g \in \mathcal{G}(X, \mathcal{P})$. Then $g$ satisfies the $R P F$ condition if and only if $P(g)>h(X)$. If $P(g)>h(X)$, then $I(g)=\{H \nu\}$.

Proof. We need only check that $P(g)=h(X)$ implies that $g$ cannot satisfy the RPF condition. To see this, suppose that $g$ does satisfy RPF. Then note that $H \nu$ is a measure which assigns positive mass to any cylinder. But $X$ is assumed to be a proper subset of $\Omega_{p}$ and so any measure in $\hat{\mathscr{K}}(X)$ must vanish on some cylinder. If $P(g)=h(X)$, then $\hat{\mathfrak{R}}(X) \subset I(g)$ and we have a contradiction.

Proposition 3.7. Let $g \in \mathcal{G}(X, \mathcal{P})$ and suppose that there exists an equilibrium state $\mu$ for $g$ such that $\mu(X)=0$. Then $\mu$ is the only such measure in $I(g)$ and hence $\mu$ is ergodic. (There may, of course, be other measures in $I(g)$.)

Proof. Suppose that there exist two distinct ergodic measures $\mu$ and $\bar{\mu}$ in $I(g)$ such that $\mu(X)=\bar{\mu}(X)=0$. Then there would have to be a cylinder set $B_{0}$ such that $B_{0} \cap \rho(X)=\varnothing$ and $\mu\left(B_{0}\right) \neq \bar{\mu}\left(B_{0}\right)$. We may assume that the length of $B_{0}$ is larger than $K_{0}$, and then define a new partition $\mathcal{P}^{\prime}=\left\{\rho(X), M_{0}^{\prime}, M_{1}^{\prime}, \ldots\right\}$ where $M_{0}^{\prime}=\left\{w: w \in B_{0}\right\}$ and $M_{i}^{\prime}=\left(M_{i-1}-B_{0}\right)$ for $i \geqslant 1$. It follows that $\mathcal{P}^{\prime}$ also satisfies conditions (a) through (e), and so we may view $g$ as being an element of $\mathcal{G}\left(X, \mathcal{P}^{\prime}\right)$. 
We now use result III from $\S 1$ and the ergodicity of $\mu$ to conclude that there exist $g_{n} \in \mathcal{G}\left(X, \mathcal{P}^{\prime}\right)$ satisfying:

(i) $g_{n} \rightarrow g$,

(ii) $I\left(g_{n}\right)=\left\{H_{n} \nu_{n}\right\}$, where $\mathcal{L}_{g_{n}} H_{n}=e^{P\left(g_{n}\right)} H_{n}$ and $\mathcal{L}^{*} \nu_{n}=e^{P\left(g_{n}\right)} \nu_{n}$ and $H_{n}$ is constant on any cylinder $B$ of length larger than $K_{0}$ such that $B \cap \rho(X)=\varnothing$,

(iii) $\lim _{n \rightarrow \infty} H_{n} \nu_{n}=\mu$.

Without loss of generality we assume that the sequence $\nu_{n}$ converges to some measure $\nu$. Then $\mathcal{L}_{g}^{*}(\nu)=e^{P(g)} \nu$ and for any cylinder $B$ such that $B \cap \rho(X)=\varnothing, z$ $\in B$ implies $\mathcal{L}_{g} H(z)=e^{P(g)} H(z)$, where $H$ is defined by $H(z)=\lim _{n \rightarrow \infty} H_{n}(z)$. Note that $H$ is only defined on $\Omega_{p}^{+}-\rho(X)$. Thus $\mu(B)=\lim H_{n}(B) \nu(B)=$ $H(B) \nu(B)$ and for any block $A$,

$$
\mu(A B)=H(A B) \nu(A B)=H(A B) K(A, B) \nu(B) .
$$

In particular, $\mu(B A B)=K(B A, B) H(B) \nu(B)$. Now let $B$ be the previously chosen cylinder set $B_{0}$ and apply a theorem of $\mathrm{Kac}[3]$ to conclude that

$$
1=\int_{B_{0}} r(z) d \mu(z)=(\Sigma \text { specific numbers }) H\left(B_{0}\right) \nu\left(B_{0}\right),
$$

where $r(z)$ denotes the first return to $B_{0}$ function and the "specific numbers" are determined entirely by $g$ and $\mathcal{P}^{\prime}$. Therefore $\mu\left(B_{0}\right)=H\left(B_{0}\right) \nu\left(B_{0}\right)$ is determined, so $\mu\left(B_{0}\right)=\bar{\mu}\left(B_{0}\right)$, and the proof is completed.

Another way of phrasing the preceding argument is that if $\mu, \bar{\mu} \in I(g)$ satisfy $\mu(X)=\bar{\mu}(X)=0$, then $\mu$ and $\bar{\mu}$ induce the same measure transformation on any cylinder $B$ such that $B \cap \rho(X)=\varnothing$. Thus $\mu=\bar{\mu}$. This can be compared to Walters results [10] concerning the Hofbauer examples.

The following corollary is an immediate consequence of the preceding proposition and Theorems 1.1 and 1.2 from $\$ 1$.

Corollary 3.8. If $g \in \mathcal{G}(X, \mathcal{P})$ and $P(g)=h(X)$, then either

(a) $I(g)=\hat{\mathscr{T}}(X)$ or

(b) $I(g)=$ closed convex hull of $\hat{\mathfrak{R}}(X)$ and the measure described in Proposition 3.7.

Furthermore, every proper subshift $X$ of $\Omega_{p}$ has functions $g_{1}, g_{2} \in \mathcal{G}(X, \mathcal{P})$ such that $I\left(g_{1}\right)$ is as in (a) and $I\left(g_{2}\right)$ is as described in (b).

In Proposition 2.2 and only there have we used the fact that if $g \in \mathcal{G}(X, \mathscr{P})$ is locally constant, then $\mathcal{L}_{g}^{*}$ has $e^{P(g)}$ as an eigenvalue for some eigenmeasure $\nu \in \mathfrak{R}^{+}$. This could have been avoided in the following way. Start with $g$ locally constant and without loss of generality assume that $g(\overline{0})=0$, where $\overline{0}$ denotes the sequence of all zeros. Let $X=\{\overline{0}\}$ and construct a partition $\mathscr{P}$ satisfying conditions (a) through (e) with the further property that $\mathscr{P}$ is fine enough so that $g \in \mathcal{G}(X, \mathcal{P})$. Then the Schauder-Tychonoff fixed-point theorem guarantees the existence of a real number $\lambda$ and a measure $\nu \in \mathfrak{R}^{+}$such that $\varrho_{g}^{*} \nu=\lambda \nu$. One then can easily show that $\lambda>1$, so $\log \lambda$ is larger than the topological entropy of $X$. Now use the proofs of $\$ 3$ (which rely on facts about $g$-measures) to conclude that $P(g)=\log \lambda$ and that the unique equilibrium state for $g$ is $\mu=H \nu$. This is then a roundabout way of establishing a 
well-known fact about the pressure of locally constant functions defined on full shifts. While this is surely not the way to establish this well-known fact, it does show how the theory of grid functions does in spirit include the facts about locally constant functions.

4. Matrix partitions. Let $X$ be a matrix subshift. In other words, there exists $a$ 0-1, $p \times p$ matrix $A$ such that

$$
X=\left\{w \in \Omega_{p}: A\left(w_{i}, w_{i+1}\right)=1 \forall i\right\} .
$$

There is a natural partition on $\Omega_{p}^{+}$associated with $X$, namely,

$$
\begin{aligned}
M_{0} & =\left\{x: A\left(x_{0}, x_{1}\right)=0\right\}, \\
M_{1} & =\left\{x: A\left(x_{0}, x_{1}\right)=1, A\left(x_{1}, x_{2}\right)=0\right\}, \\
& \vdots \\
M_{n} & =\left\{x: \sum_{k=0}^{n-1} A\left(x_{k}, x_{k+1}\right)=n, A\left(x_{n}, x_{n+1}\right)=0\right\}, \\
& \vdots \\
\rho(X) & =\left\{x: A\left(x_{i}, x_{i+1}\right)=1 \forall i \geqslant 0\right\} .
\end{aligned}
$$

We will assume that $A(i, j)=1$ implies that there exists $k$ such that $A(k, i)=1$, that is, if a symbol has a successor, it also has a predecessor. It follows that the above $\rho(X)$ coincides with $\rho(X)$ in $\S 1$. Moreover, it is easy to check that $\mathscr{P}=$ $\left\{\rho(X), M_{0}, M_{1}, \ldots\right\}$ satisfies conditions (a) through (e) from $\$ 1$. Consequently all the results in the preceding sections apply here. Under mild assumptions on $A, P(g)$ and $I(g)$ for $g \in \mathcal{S}(X, \mathcal{P})$ can be studied analytically; and the remaining sections are devoted to describing more explicit information about $P(g)$ and $I(g)$ in this context.

Let $g \in \mathcal{G}(X, \mathcal{P})$ and suppose $\nu$ is an eigenmeasure for $g$, i.e. $\mathcal{L}_{g}^{*} \nu=\lambda \nu$ for some $\lambda>0$. Using $E_{i}=\left\{x: x_{0}=i\right\}$ as in $\S 2$, define the following column vectors:

$$
\begin{gathered}
\xi=\left(\begin{array}{c}
\nu\left(E_{1}\right) \\
\vdots \\
\nu\left(E_{p}\right)
\end{array}\right), \quad \nu_{i}=\left(\begin{array}{c}
\nu\left(M_{i} \cap E_{1}\right) \\
\vdots \\
\nu\left(M_{i} \cap E_{p}\right)
\end{array}\right), \quad i>0, \\
\nu_{\infty}=\left(\begin{array}{c}
\nu\left(\rho(X) \cap E_{1}\right) \\
\vdots \\
\nu\left(\rho(X) \cap E_{p}\right)
\end{array}\right) .
\end{gathered}
$$

Let $s_{k}=\sum_{j=0}^{k} a_{j}$, and let $J$ be the $p \times p$ matrix of all ones.

LEMMA 4.1. The following formulas hold:

(1) $\lambda \nu_{0}=e^{a_{0}}(J-A) \xi$,

(2) $\lambda \nu_{k}=e^{a_{k}} A \nu_{k-1}$,

(3) $\nu_{k}=e^{s_{k}} A^{k}(J-A) \xi / \lambda^{k+1}$,

(4) $\xi=\nu_{\infty}+\sum_{k=0}^{\infty} e^{s_{k}} A^{k}(J-A) \xi / \lambda^{k+1}$. 
Proof. First note that (3) is an obvious consequence of (1) and (2), and (4) follows from (3) because

$$
\xi=\nu_{\infty}+\sum_{k=0}^{\infty} \nu_{k} .
$$

To prove (1) we compute $\lambda \nu\left(M_{0} \cap E_{i}\right)$ as follows:

$$
\begin{aligned}
\lambda \nu\left(M_{0} \cap E_{i}\right) & =\nu\left(\mathcal{L} \chi_{M_{0} \cap E_{i}}(w)\right) \\
& =\nu\left(\sum_{j=1}^{p} e^{g(j w)} \chi_{M_{0} \cap E_{i}}(j w)\right) \\
& =\nu\left(e^{g(i w)} \chi_{M_{0}}(i w)\right) \\
& =e^{a_{0}} \nu\left(\sum_{j=1}^{p}[1-A(i, j)] \chi_{E_{j}}(w)\right) \\
& =e^{a_{0}} \sum_{j=1}^{p}[1-A(i, j)] \nu\left(E_{j}\right) .
\end{aligned}
$$

Similarly

$$
\begin{aligned}
\lambda \nu\left(M_{k} \cap E_{i}\right) & =\nu\left(e^{g(i w)} \chi_{M_{k}}(i w)\right) \\
& =e^{a_{k}} \nu\left(\sum_{j=1}^{p} A(i, j) \chi_{M_{k-1} \cap E_{j}}(w)\right) \\
& =e^{a_{k}} \sum_{j=1}^{p} A(i, j) \nu\left(M_{k-1} \cap E_{j}\right)
\end{aligned}
$$

and this establishes (2).

Proposition 4.2. If $\nu_{\infty} \neq 0$, then $\nu_{\infty}$ is an eigenvector of $A$ belonging to $\lambda$, i.e., $A \nu_{\infty}=\lambda \nu_{\infty}$.

Before proving this we observe that

$$
\begin{aligned}
\mathcal{L} \chi_{E_{i}}(w)= & e^{g(i w)}=e^{a_{0}} \sum_{j=1}^{p}[1-A(i, j)] \chi_{E_{j}}(w) \\
& +\sum_{k=1}^{\infty} e^{a_{k}} \sum_{j=1}^{p} A(i, j) \chi_{M_{k-1} \cap E_{j}}(w) \\
& +\sum_{j=1}^{p} A(i, j) \chi_{\rho(X) \cap E_{j}}(w)
\end{aligned}
$$


and by the Monotone Convergence Theorem

$$
\begin{aligned}
\lambda \nu\left(E_{i}\right)= & e^{a_{0}} \sum_{j=1}^{p}[1-A(i, j)] \nu\left(E_{j}\right) \\
& +\sum_{k=1}^{\infty} e^{a_{k}} \sum_{j=1}^{p} A(i, j) \nu\left(M_{k-1} \cap E_{j}\right) \\
& +\sum_{j=1}^{p} A(i, j) \nu\left(\rho(X) \cap E_{j}\right) .
\end{aligned}
$$

Proof. Using the above formula we can write

$$
\begin{aligned}
\lambda\left(\sum_{k=0}^{\infty} \nu_{k}+\nu_{\infty}\right) & =\lambda \xi=e^{a_{0}}(J-A) \xi+\sum_{k=0}^{\infty} e^{a_{k}} A \nu_{k-1}+A \nu_{\infty} \\
& =\lambda \nu_{0}+\sum_{k=1}^{\infty} \lambda \nu_{k}+A \nu_{\infty}
\end{aligned}
$$

with the last inequality following from (1) and (2). Hence by cancellation $\lambda \nu_{\infty}=A \nu_{\infty}$.

The topological entropy of $X$ is $\log \alpha$ where $\alpha$ is the maximal eigenvalue of $A$. It follows from the above proposition that $\nu_{\infty}=0$ when $\lambda>\alpha$. (We gave a different proof of this in §2.)

Consider the matrix valued analytic function

$$
\tilde{g}(z)=\sum_{k=0}^{\infty} \frac{e^{s_{k}}}{\lambda^{k+1}} z^{k+1} A^{k}(J-A) .
$$

Since every entry in each term of the series is positive and each component of $\xi$ is positive, it follows from (4) that the radius of convergence is at least one.

Moreover, equation (4) from Lemma 4.1 can now be written

$$
\xi=\nu_{\infty}+\tilde{g}(1) \xi
$$

This suggests the possibility of using the analytic function

$$
G(z)=\sum_{k=0}^{\infty} \frac{e^{s_{k}}}{\alpha^{k+1}} z^{k+1} A^{k}(J-A),
$$

which depends only on $A$ and $g$, to study $I(g)$. A brief synopsis of the results of this program is presented in the remaining sections, and the complete proofs can be found in the author's Technical Report [5]. A key ingredient in these arguments is the structure of irreducible matrices for which Chapter 2 of Varga [7] is a satisfactory source.

5. Block irreducible matrices. Let $A$ be a nonzero $p \times p$ matrix. We will say $A$ is block irreducible if there exists a permutation matrix $P$ such that

$$
P A P^{t}=\operatorname{diag}\left\{R_{1}, \ldots, R_{q}\right\}
$$

and each $R_{i}$ is a square matrix which is either irreducible or zero. Without loss of generality we can assume that $R_{i} \neq 0$ for $1 \leqslant i \leqslant q-1$. As before let $\alpha$ be the maximum positive real eigenvalue and note that $\alpha$ equals the spectral radius of $A$. 
Let $\left\{a_{n}\right\}$ be a sequence of real numbers converging to 0 , and set

$$
G(z)=\sum_{k=0}^{\infty} \frac{e^{s_{k}}}{\alpha^{k+1}} z^{k+1} A^{k}(J-A)
$$

where $s_{k}=\sum_{i=0}^{k} a_{i}$. The sequences $\left\{e^{s_{k}}\right\}$ which arise this way are precisely the positive real sequences $\left\{b_{k}\right\}$ such that

$$
\lim _{k \rightarrow \infty} \frac{b_{k+1}}{b_{k}}=1
$$

THEOREM 5.1. If $A$ is a block irreducible 0-1 matrix not equal to $J$, then the radius of convergence of $G(z)$ is 1 .

Proof. Use the structure of irreducible matrices and the ratio test.

Henceforth we will assume that $A$ is a block-irreducible $0-1$ matrix not equal to $J$. We will use the notation $z=r e^{i \theta}$. Note that for $0<r<1$ and $\theta=0, G(r) \neq 0$ and $G(r) \geqslant 0$. We define a real valued function $\eta$ on $(0,1)$ by setting $\eta(r)$ equal to the maximum real eigenvalue of $G(r)$. Clearly the function $\eta(r)$ is strictly increasing on $(0,1)$ and there exists a unique $\xi(r)>0$ in $\mathbf{R}^{p}$ such that $\sum_{i=1}^{p} \xi_{i}(r)=1$ and $G(r) \xi(r)=\eta(r) \xi(r)$.

Now let $X$ be the matrix subshift of $\Omega_{p}$ determined by $A$ and let $g(x)=$ $\sum_{n=0}^{\infty} a_{n} \chi_{M_{n}} \in \mathcal{G}(X, \mathcal{P})$.

THEOREM 5.2. Let $\lambda$ be a real number. The following are equivalent:

(a) $\log \lambda=P(g)$.

(b) There exists $\nu \in \mathfrak{T}$ such that $\mathcal{L}^{*} \nu=\lambda \nu$.

(c) $\lambda=\alpha / \min \left[\{1\} \cup \eta^{-1}(1)\right]$.

Proof. It was shown in Proposition 2.2 that (a) implies (b). If (b) implies (c), then (a) implies (c) and (c) implies (a) becomes a triviality. The rest of the proof is devoted to (b) implies (c).

Suppose $\mathcal{L}^{*} \nu=\lambda \nu$ and recall (4) from $\S 4$. It can be written

$$
\xi=\nu_{\infty}+G(\alpha / \lambda) \xi \text {. }
$$

It follows that $\alpha / \lambda \leqslant 1$ from Theorem 5.1. If $\lambda>\alpha$, then $\nu_{\infty}=0, \xi=G(\alpha / \lambda) \xi$, and $\eta(\alpha / \lambda)=1$.

COROLLARY 5.3. The following are equivalent:

(a) $\nu_{\infty}=0$ for some $\nu \in \mathbb{R}$ satisfying $\mathfrak{L}^{*} \nu=\lambda \nu, \lambda$ real.

(b) $\eta(1) \geqslant 1$.

(c) $\nu_{\infty}=0$ for all $\nu \in \mathfrak{R}$ satisfying $\mathfrak{L}^{*} \nu=\lambda \nu, \lambda$ real.

Proof. Since $\mathcal{L}^{*} \nu=\lambda \nu$ implies $\log \lambda=P(g), \tilde{g}(z)=G(\alpha z / \lambda)$ is completely determined by $g$ and the result follows from

$$
\xi=\nu_{\infty}+\tilde{g}(1) \xi
$$

because the maximal eigenvalue and its positive eigenvectors are the optimal solution of $A x \leqslant \rho x, x>0$. 
The property that $\eta(1) \geqslant 1$ will be useful later and we call such a function nonsingular. (If $\nu_{\infty} \neq 0$, then $\nu \mid \rho(X)$ can be thought of as a singular part of $\nu$.) When $\nu_{\infty}=0$, equation (4) is an eigenvalue equation. Since $\sum_{i=1}^{p} \xi_{i}=1$, $\xi$ is uniquely determined by $g$; that is, $\xi$ is independent of $\nu$ because of the nature of the matrix $\tilde{g}(1)$ which is completely determined by $g$.

6. Determination of eigenfunctions. As in the preceding section let $X$ be a matrix subshift given by a block irreducible matrix $A$, and let $g=\sum_{n=0}^{\infty} a_{n} \chi_{M_{n}} \in \mathcal{G}(X, \mathscr{P})$. If $\log \lambda=P(g)>\log \alpha=h(X)$, then by Theorem 3.6 we know that $g$ is RPF and there exists a positive continuous eigenfunction $H$ for $\mathcal{L}_{g}$. The purpose of this section is to explicitly determine $H$ in this concrete setting, that is, to compute $\lim _{n \rightarrow \infty} \lambda^{-n} \mathcal{L}^{n} 1$. The technique is a matrix analogue of a proof given by Hofbauer $[2, \S 2]$; the essential difference and added difficulty is replacing the use of a standard renewal theorem for sequences by a calculation with matrix valued analytic functions of a single complex variable.

We will let $\mathcal{L}$ act componentwise on continuous vector valued functions defined on $\Omega_{p}^{+}$, and we will let

$$
\Psi(x)=\left(\begin{array}{c}
\chi_{E_{1}}(x) \\
\vdots \\
\chi_{E_{p}}(x)
\end{array}\right)
$$

For $n \geqslant 1$ and $1 \leqslant i \leqslant p$ let $\beta$ be any $n$-block ending with $i$ and occurring in $X$ and define a continuous function on $\Omega_{p}^{+}$by $W_{n, i}(x)=\exp \left(S_{n-1} g(\beta x)\right)$ which is independent of the choice of $\beta$. (We will use the notation $S_{k} g=\sum_{i=0}^{k} g \circ \sigma^{i}$.) If no such $\beta$ exists, set $W_{n, i}(w) \equiv 0$. Then define the matrix valued function $\mho_{n}(x)$ by

$$
\mathscr{W}_{n}(x)=A^{n-1} \operatorname{diag}\left\{W_{n, 1}(x), \ldots, W_{n, p}(x)\right\} A .
$$

A direct calculation shows that for $n \geqslant 1$

$$
\mathcal{W}_{n}(x) \Psi(x)= \begin{cases}e^{-s_{k}} e^{s_{k+n}} A^{n} \Psi(x) & \text { if } x \in M_{k}, \\ A^{n} \Psi(x) & \text { if } x \in \rho(X),\end{cases}
$$

and

$$
\mathcal{L}\left(\mathscr{W}_{n} \Psi\right)(x)=e^{s_{n}} A^{n}(J-A) \Psi(x)+\mathcal{W}_{n+1}(x) \Psi(x) .
$$

Let $Q_{0}(x)=\Psi(x)$ and $Q_{k}(x)=\mathcal{L}^{k} \Psi$. Then

$$
\begin{aligned}
\mathscr{Q}_{1}(x) & =\mathcal{L}(\Psi)(x)=\left(\begin{array}{c}
e^{g(1 x)} \\
\vdots \\
e^{g(p x)}
\end{array}\right) \\
& =e^{a_{0}}(J-A) \Psi(x)+\operatorname{diag}\left\{e^{g(1 x)}, \ldots, e^{g(p x)}\right\} A \Psi(x) \\
& =e^{a_{0}}(J-A) \mathbb{Q}_{0}(x)+\mathscr{W}_{1}(x) \Psi(x) .
\end{aligned}
$$

Thus by induction

$$
\mathbb{Q}_{n}(x)=\sum_{k=1}^{n} e^{s_{k-1} A^{k-1}(J-A) \mathbb{Q}_{n-k}}+\mathscr{W}_{n}(x) \Psi(x) .
$$


If we set $\Re_{n}(x)=\lambda^{-n} \mathscr{Q}_{n}$, then this formula can be written as follows:

$$
\mathscr{B}_{n}=\sum_{k=1}^{n} \frac{e^{s_{k-1}}}{\lambda^{k}} A^{k-1}(J-A) \mathscr{B}_{n-k}+\lambda^{-n} \mho_{n}(x) \Psi(x) .
$$

Notice that the coefficient of $\mathscr{B}_{n-k}$ in this expression is also the coefficient of $z^{k}$ in $\tilde{g}(z)=G(\alpha z / \lambda)$, which is analytic in $|z|<\lambda / \alpha$. The next few propositions will allow us to take advantage of this connection.

We begin by observing that $\tilde{g}(z)=G(\alpha z / \lambda)$ is the same $\tilde{g}(z)$ appearing in $\S 4$ and that (4) can be written as $\xi=\tilde{g}(1) \xi$ because we are assuming $\lambda>\alpha$. Hence $I-\tilde{g}(1)$ is a singular matrix.

Proposition 6.1. For $|z| \leqslant 1$ and $z \neq 1, I-\tilde{g}(z)$ is nonsingular.

Proof. The proof is based on the following facts:

(i) The spectral radius of $\tilde{g}(1)$ is 1 .

(ii) If a single entry of a primitive matrix is increased, then the spectral radius increases.

(iii) If $f(z)=\sum_{n=0}^{\infty} c_{n} z^{n}$ for $|z|<r, c_{n} \geqslant 0$, and two consecutive $c_{n}$ 's are positive, then $|f(z)|<f(|z|)$ for $z \neq|z|<r$.

Let $\phi(z)=(I-\tilde{g}(z))^{-1}$. By Proposition $6.1 \phi(z)$ is analytic in the unit disk and $z=1$ is the only singular point on the boundary. There exist $p \times p$ real matrices $U_{n}$ such that for $|z|<1$

$$
\phi(z)=\sum_{n=0}^{\infty} z^{n} U_{n}
$$

Proposition 6.2. The function $(z-1) \phi(z)$ is analytic on $\{z:|z|<1+\delta\}$ for some $\delta>0$.

Proof. It suffices to show that

$$
\lim _{z \rightarrow 1} \frac{\operatorname{det}(I-\tilde{g}(z))}{z-1} \neq 0 .
$$

Define $\tilde{g}_{1}(z)$ by $\tilde{g}(z)=z \tilde{g}_{1}(z)$ and note that 1 is a simple root $\operatorname{of} \operatorname{det}\left(\lambda I-\tilde{g}_{1}(1)\right)$. Use the implicit function theorem to write $\operatorname{det}\left(\lambda I-\tilde{g}_{1}(z)\right)=(\lambda-k(z)) K(\lambda, z)$ and differentiate

$$
\operatorname{det}\left(I-z \tilde{g}_{1}(z)\right)=(1-z k(z)) z^{p-1} K(1 / z, z) .
$$

COROLlaRY 6.3. The sequence of matrices $\left\{U_{n}\right\}$ is convergent.

Proof. For $|z|<1$ we have

$$
(z-1) \phi(z)=-U_{0}+\sum_{n=1}^{\infty} z^{n}\left(U_{n-1}-U_{n}\right) .
$$

By the proposition we just proved, this formula must hold for $|z|<1+\delta$ for some $\delta>0$. In particular, for $z=1$ we have

$$
\lim _{z \rightarrow 1}(z-1) \phi(z)=\lim _{n \rightarrow \infty}-U_{n}
$$


The matrix $U=\lim _{n \rightarrow \infty} U_{n}$ satisfies two useful equations. First letting $z$ tend to 1 in the equation

$$
(1-z) \phi(z)[I-\tilde{g}(z)]=(1-z) I
$$

yields $U \tilde{g}(1)=U$. Similarly $\tilde{g}(1) U=U$, and hence

$$
U \tilde{g}(1)=U=\tilde{g}(1) U .
$$

Also from $\phi(z)[I-\tilde{g}(z)]=I$ we get

$$
(1-z) \phi(z) \frac{[\tilde{g}(z)-\tilde{g}(1)]}{z-1} \xi=\xi
$$

by using $\tilde{g}(1) \xi=\xi=I \xi$. Again let $z$ approach 1 to get

$$
U \tilde{g}^{\prime}(1) \xi=\xi \text {. }
$$

Clearly the set of nonnegative matrices satisfying

$$
V \tilde{g}(1)=V=\tilde{g}(1) V
$$

is a ray emanating from the origin in the space of all $p \times p$ real matrices. Therefore, $U$ is uniquely determined by equations (5) and (6).

For convenience let

$$
V_{n}=\frac{e^{s_{n}}}{\lambda^{n+1}} A^{n}(J-A)
$$

so that $\tilde{g}(z)=\sum_{n=1}^{\infty} z^{n+1} V_{n}$. Since $\phi(z)=(I-\tilde{g}(z))^{-1}$ can be written $\phi(z)=I+$ $\phi(z) \tilde{g}(z)$, we have

$$
U_{0}=I, \quad U_{1}=U_{0} V_{0}, \quad U_{2}=U_{1} V_{0}+U_{0} V_{1},
$$

and $U_{n}=U_{n-1} V_{0}+\cdots+U_{0} V_{n-1}$.

We can now return to the computation of the eigenfunctions. First we rewrite $(* *)$ as

$$
\Re_{n}=\sum_{i=0}^{n-1} V_{n-1-i} \Re_{i}+\frac{1}{\lambda^{n}} \mathscr{W}_{n} \Psi
$$

and form

$$
\sum_{j=0}^{n} U_{n-j} \mathscr{B}_{j}=U_{n} \mathscr{B}_{0}+\sum_{j=1}^{n} U_{n-j}\left(\sum_{i=0}^{j-1} V_{j-1-i} \mathscr{B}_{i}+\frac{1}{\lambda^{j}} \mho_{j} \Psi\right)
$$

Interchanging the order of summation and using the above formula, we find that

$$
\mathscr{B}_{n}=\left(\sum_{j=1}^{n} \frac{1}{\lambda^{j}} U_{n-j} \mathscr{w}_{j}\right) \Psi
$$

Let

$$
\Gamma_{n}=\sum_{j=1}^{n} \frac{1}{\lambda^{j}} U_{n-j} \text { w }_{j}
$$


and define a matrix valued function $\mathcal{H}$ on $\Omega_{p}^{+}$by

$$
\mathcal{H}(x)= \begin{cases}e^{-s_{k}} U \sum_{m=1}^{\infty} \frac{e^{s_{k+m}}}{\lambda^{m}} A^{m}, & \text { for } x \in M_{k}, \\ U \sum_{m=1}^{\infty} \frac{1}{\lambda^{m}} A^{m}, & \text { for } x \in \rho(X) .\end{cases}
$$

THEOREM 6.4. The sequence of functions $\left\{\Gamma_{u}\right\}$ converges uniformly on $\Omega_{p}^{+}$to $\mathcal{H}$.

The proof of Theorem 6.4 is similar to Hofbauer's proof of convergence [ 2, pp. 228 and 229].

COROLlaRY 6.5. The sequence of functions $\lambda^{-n} \mathcal{L}^{n}(1)$ converges uniformly on $\Omega_{p}^{+}$to $H(x)=(1, \ldots, 1) \mathcal{H}(x) \Psi(x), H(x)>0$ on $\Omega_{p}^{+}$and $\mathcal{L}(H)=\lambda H$.

Proof. Just observe that

$$
\lambda^{-n} \mathcal{L}^{n}(1)=(1, \ldots, 1) \mathscr{B}_{n}=(1, \ldots, 1) \Gamma_{n} \Psi .
$$

7. Bifurcation of equilibrium states. Let $g \in \mathcal{G}(X, \mathscr{P})$ and again consider the series

$$
G(z)=\sum_{n=0}^{\infty} \frac{e^{s_{n}}}{\alpha^{n+1}} z^{n+1} A^{n}(J-A) .
$$

If some entry diverges when $z=1$, then obviously $g$ is an RPF function. So we assume $G(1)$ is finite and consider $\eta(1)$, the maximal eigenvalue of the matrix $G(1)$. If $\eta(1)>1$, then $g$ is still an RPF function. The next two propositions show that $\eta(1)=1$ is the interesting case.

Proposition 7.1. If $\eta(1)<1$, then $I(g)=\hat{\mathfrak{R}}(X)$.

Proof. Suppose $\mu \in I(g)$ and $\mu \notin \hat{\mathfrak{R}}(X)$. Then clearly $\mu(X)<1$ because $P(g)$ $=\log \alpha$. So there exists $M_{k}$ such that $\mu\left(M_{k}\right)>0$, where $\mu$ also denotes its own projection on $\Re^{+}$. Consider $g_{\tau}=g+\tau \chi_{M_{k}}$. Then

$$
G_{\tau}(z)=\sum_{j=0}^{k-1} \frac{e^{s_{j}}}{\alpha^{j+1}} z^{j+1} A^{j}(J-A)+e^{\tau} \sum_{j=k}^{\infty} \frac{e^{s_{j}}}{\alpha^{j+1}} z^{j+1} A^{j}(J-A)
$$

and for small positive $\tau$ we still have $\eta_{\tau}(1)<1$. But this produces the contradiction

$$
\log \alpha=P\left(g_{\tau}\right) \geqslant \mu\left(g_{\tau}\right)+s(\mu)>\mu(g)+s(\mu)=P(g)=\log \alpha .
$$

PROPOSITION 7.2. Let $k \geqslant 0$ and set $g_{\tau}=g+\tau \chi_{M_{k}}$. If $\eta(1)=1$, then $g_{\tau}$ is an $R P F$ function for $\tau>0$ and $I\left(g_{\tau}\right)=\hat{\mathfrak{N}}(x)$ for $\tau<0$.

Proof. This is obvious from the calculation of $G_{\tau}$ in the previous proof.

We will say the function $g \in \mathcal{G}(X, \mathcal{P})$ is at bifurcation if $\eta(1)=1$. In this section we will characterize the structure of $I(g)$ for $g$ at bifurcation in terms of the behavior of $G^{\prime}(1)$. Notice that for $g$ at bifurcation $P(g)=\log \alpha$, i.e. $\lambda=\alpha$ and hence $\tilde{g}(z)=G(\alpha z / \lambda)=G(z)$ and $\hat{\Re}(X) \subset I(g)$.

In fact, $g$ is at bifurcation if and only if $g$ is nonsingular and $P(g)=\log \alpha$. Moreover, $I-\tilde{g}(z)$ is clearly nonsingular for $|z|<1$. 
Throughout the rest of this section we will be dealing with uniformly convergent sequences $\left\{g_{n}\right\}$ in $\mathcal{G}(X, \mathcal{P})$ and the following notation will be used:

$$
\begin{gathered}
g_{n}=\sum_{k=0}^{\infty} a_{k}(n) \chi_{M_{k}}, \quad s_{k}(n)=\sum_{i=0}^{k} a_{i}(n), \\
P\left(g_{n}\right)=\log \lambda_{n}, \quad \tilde{g}_{n}(z)=G_{n}\left(\alpha z / \lambda_{n}\right), \\
G_{n}(z)=\sum_{k=0}^{\infty} \frac{\exp \left(s_{k}(n)\right)}{\alpha^{k+1}} z^{k+1} A^{k}(J-A),
\end{gathered}
$$

etc. In addition, $\nu_{n} \in \mathfrak{R}^{+}$will always satisfy $\mathcal{L}^{*} \nu_{n}=\lambda_{n} \nu_{n}$. Throughout this section we will assume that the sequence $\left\{g_{n}\right\} \subset \mathcal{G}(X, \mathscr{P})$ converges uniformly to $g$.

As in the previous section all the notation without the subscript $n$ will apply to $g$.

For $|z|<1$ we know that

$$
G^{\prime}(z)=\sum_{n=0}^{\infty} \frac{(n+1) e^{s_{n}}}{\alpha^{n+1}} z^{n} A^{n}(J-A) .
$$

This formula also makes sense for $z=1$.

Proposition 7.3. The following statements hold:

(a) If $g$ is nonsingular, then $\xi_{n}$ converges to $\xi$.

(b) If $g_{n}$ is nonsingular for all $n$, then $\tilde{g}_{n}(z)$ converges to $\tilde{g}(z), \tilde{g}_{n}^{\prime}(z)$ converges to $\tilde{g}^{\prime}(z)$ on $|z|<1$ and there exists a subsequence which converges uniformly on compact subsets.

(c) $g$ is nonsingular if and only if $\tilde{g}_{n}(1)$ converges to $\tilde{g}(1)$.

The proofs of parts (a) and (c) are just matrix arguments and (b) uses normal families.

From now on we will also assume that the function $g$ is at bifurcation, and $\lambda_{n}>\alpha$ for all $n$. Note that $\lambda_{n}>\alpha$ implies $g_{n}$ is RPF and nonsingular. Let

$$
\left[I-g_{n}(z)\right]^{-1}=\varphi_{n}(z)=\sum_{k=0}^{\infty} U_{k}(n) z^{k}
$$

and

$$
U(n)=\lim _{k \rightarrow \infty} U_{k}(n)
$$

It is to see that $\tilde{g}^{\prime}(1) \leqslant \lim _{n \rightarrow \infty} \tilde{g}_{n}^{\prime}(1)$ and using (6) it can be shown that $\left\{U(n) \tilde{g}_{n}^{\prime}(1)\right\}$ is bounded. These facts are used to prove

Proposition 7.4. The sequence $\{U(n)\}$ is bounded. Moreover, if $U(n)$ converges to $U \neq 0$, then $\left\{\tilde{g}_{n}^{\prime}(1)\right\}$ is bounded.

Proposition 7.5. The following statements hold:

(a) If $\tilde{g}^{\prime}(1)$ is not finite, then $\lim _{n \rightarrow \infty} U(n)=0$.

(b) If $\tilde{g}^{\prime}(1)$ is finite, then $U(n)$ converges to some $U \neq 0$ if and only if $\tilde{g}_{n}^{\prime}(1)$ converges to $\tilde{g}^{\prime}(1)$. 
(c) If $\tilde{g}^{\prime}(1)$ is finite and $U(n)$ converges to $U \neq 0$, then

$$
U=\lim _{r \rightarrow 1^{-}}(1-r) \varphi(r)
$$

as well as being the unique solution of $U \tilde{g}(1)=U=\tilde{g}(1) U$ and $U \tilde{g}^{\prime}(1) \xi=\xi$.

(d) If $g^{\prime}(1)$ is finite, then the only possible cluster points for the sequence $\{U(n)\}$ are 0 and $\lim _{r \rightarrow 1^{-}}(1-r) \varphi(r)$.

Proof. Suppose $\tilde{g}^{\prime}(1)$ is finite and $U(n)$ converges to $U$. Set

$$
\psi(x)=\sum_{k=0}^{\infty}(k+1) \chi_{M_{k}}, \quad \nu_{n}^{\prime}=\psi(x) \nu_{n} \quad \text { and } \quad \nu^{\prime}=\psi(x) \nu .
$$

It follows that

$$
\left(\begin{array}{c}
\nu_{n}^{\prime}\left(E_{1}\right) \\
\vdots \\
\nu_{n}^{\prime}\left(E_{p}\right)
\end{array}\right)=\sum_{k=0}^{\infty}(k+1)\left(\begin{array}{c}
\nu_{n}\left(M_{k} \cap E_{1}\right) \\
\vdots \\
\nu_{n}\left(M_{k} \cap E_{p}\right)
\end{array}\right)=\tilde{g}_{n}^{\prime}(1) \xi_{n} .
$$

If $U \neq 0$, then $\nu_{n}^{\prime}\left(\Omega_{p}^{+}\right)$are bounded and without loss of generality $\nu_{n}^{\prime}$ converges weakly to $\nu^{\prime \prime}$. Clearly $\nu^{\prime \prime}\left(M_{k} \cap E_{i}\right)=\nu^{\prime}\left(M_{k} \cap E_{i}\right)$ which forces $\tilde{g}_{n}^{\prime}(1) \xi_{n}$ to converge to $\tilde{g}^{\prime}(1) \xi$. If $Q$ is any cluster point of $\tilde{g}_{n}^{\prime}(1)$, then $Q \xi=\tilde{g}^{\prime}(1) \xi$ and $Q \geqslant \tilde{g}^{\prime}(1)$ which implies $Q=g^{\prime}(1)$. This proves half of $(b)$; the rest of the implications follow directly from the facts at hand.

Proposition 7.6. If the sequence $\{U(n)\}$ is convergent with $\lim _{n \rightarrow \infty} U_{n}=U$, then the sequence of matrix valued functions $\left\{\mathcal{H}_{n}(x)\right\}$ is pointwise convergent off of $\rho(X)$ to

$$
\mathcal{H}(x)=e^{-s_{k}} U \sum_{m=1}^{\infty} \frac{e^{s_{k+m}}}{\alpha^{m}} A_{m} .
$$

Proof. Set

$$
h_{n}(k)=\sum_{m=1}^{\infty} \frac{\exp \left(s_{k+m}(n)\right)}{\lambda_{n}^{m}} A^{m} .
$$

Then $\tilde{g}_{n}(1)$ is convergent if and only if $h_{n}(k)$ is convergent and for $x \in M_{k}$

$$
\mathcal{H}_{n}(x)=\exp \left(-s_{k}(n)\right) U(n) h_{n}(k) .
$$

COROLlaRY 7.7. If $\{U(n)\}$ is convergent, then the sequence of eigenfunctions $\left\{H_{n}(x)\right\}$ is pointwise convergent off $\rho(X)$.

COROllaRY 7.8. If $g^{\prime}(1)$ is finite and $\tilde{g}_{n}^{\prime}(1)$ converges to $\tilde{g}^{\prime}(1)$, then $H_{n}$ converges pointwise off $\rho(X)$ to a positive continuous function $H$ on $\Omega_{p}^{+} \backslash \rho(X)$ such that $\mathcal{L} H(x)=\alpha H(x)$ for all $x \notin \rho(X)$.

The following theorem can be proved by applying the results of this section together with Theorem 2.4 and Proposition 3.7 to suitable sequences $\left\{g_{n}\right\}$. 
THEOREM 7.9. Suppose $g$ is at bifurcation.

(a) If $G^{\prime}(1)$ is not finite, then $I(g)=\hat{\mathfrak{K}}(X)$.

(b) If $G^{\prime}(1)$ is finite, then there exists a unique $\mu_{0} \in I(g)$ such that $\mu_{0}(X)=0$ and $I(g)$ is the convex hull of $\hat{\Re}(X)$ and $\mu_{0}$. Furthermore, the projection of $\mu_{0}$ on $\mathfrak{R}^{+}$is $H \nu$ where $H=(1, \ldots, 1) \mathcal{H} \xi$,

$$
\mathcal{H}(x)=e^{-s_{k}} U \sum_{m=1}^{\infty} \frac{e^{s_{k+m}}}{\alpha^{m}} A^{m}
$$

for $x \in M_{k}$, and $\nu$ is the unique element of $\Re^{+}$such that $\mathfrak{L}_{g}^{*} \nu=\alpha \nu$.

\section{REFERENCES}

1. M. Fisher, The theory of condensation and the critical point, Physics 3 (1967), 255-283.

2. F. Hofbauer, Examples for the nonuniqueness of the equilibrium state, Trans. Amer. Math. Soc. 228 (1977), 223-241.

3. M. Kac, On the notion of recurrence in discrete stochastic processes, Bull. Amer. Math. Soc. 53 (1947), $1002-1010$

4. N. Markley and M. Paul, Equilibrium states supported on periodic orbits, University of Maryland Technical Report TR 78-50.

5. __ Equilibrium states of grid functions, University of Maryland Technical Report TR 80-73.

6. D. Ruelle, Thermodynamic formalism, Addison-Wesley, Reading, Mass., 1978.

7. R. Varga, Matrix iterative analysis, Prentice-Hall, Englewood Cliffs, N.J., 1962.

8. P. Walters, Ruelle's operator theorem and g-measures, Trans. Amer. Math. Soc. 214 (1975), 375-387.

9. , A variational principle for the pressure of continuous transformations, Amer. J. Math. 97 (1976), 937-971.

10. Invariant measures and equilibrium states for some mappings which expand distances, Trans. Amer. Math. Soc. 236 (1978), 121-153.

Department of Mathematics, University of Maryland, College Park, Maryland 20742

Department of Mathematics, University of Maryland, Baltimore County, Catonsville, MARYLAND 21228 